\title{
Les lettres de rémission des ducs de Bourgogne. Étude sur les normativités sociales, politiques et juridiques
}

Thèse de doctorat en histoire médiévale, sous la direction de Bruno Lemesle, université de Bourgogne-Franche-Comté, soutenue le 30 novembre 2018

\section{Rudi Beaulant}

\section{(2) OpenEdition \\ 12 Journals \\ Édition électronique \\ URL : https://journals.openedition.org/cem/16832 \\ DOI : $10.4000 /$ cem. 16832 \\ ISSN : 1954-3093 \\ Éditeur \\ Centre d'études médiévales Saint-Germain d'Auxerre}

Référence électronique

Rudi Beaulant, «Les lettres de rémission des ducs de Bourgogne. Étude sur les normativités sociales, politiques et juridiques », Bulletin du centre d'études médiévales d'Auxerre I BUCEMA [En ligne], 23.2 |

2019, mis en ligne le 31 janvier 2020, consulté le 22 septembre 2022. URL : http:// journals.openedition.org/cem/16832 ; DOI : https://doi.org/10.4000/cem.16832

Ce document a été généré automatiquement le 22 septembre 2022.

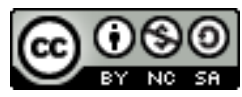

Creative Commons - Attribution - Pas d'Utilisation Commerciale - Partage dans les Mêmes Conditions 4.0 International - CC BY-NC-SA 4.0

https://creativecommons.org/licenses/by-nc-sa/4.0/ 


\section{Les lettres de rémission des ducs de Bourgogne. Étude sur les normativités sociales, politiques et juridiques}

Thèse de doctorat en histoire médiévale, sous la direction de Bruno Lemesle, université de Bourgogne-Franche-Comté, soutenue le 30 novembre 2018

Rudi Beaulant

1 Les lettres de rémission ont été beaucoup étudiées depuis la monumentale thèse d'État de C. Gauvard publiée en $1991^{1}$. Après avoir fait l'objet d'investigations par des historiens du droit et des historiens qui s'intéressaient à leur aspect «folklorique » dans la première moitié du $\mathrm{xx}^{\mathrm{e}}$ siècle ${ }^{2}$, elles suscitent de nombreux travaux depuis les renouvellements des problématiques liées à l'histoire de la

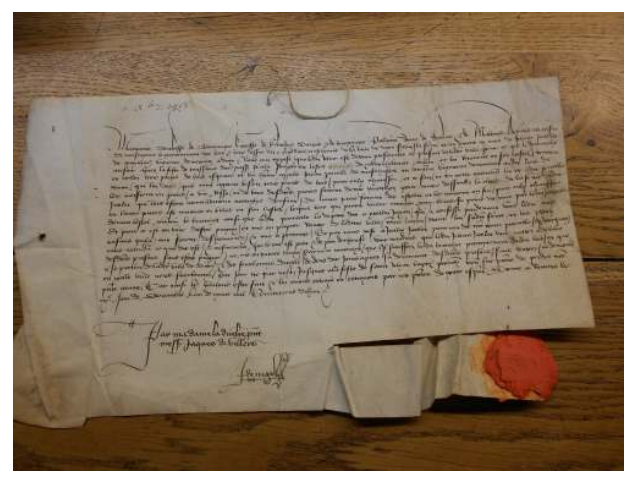
justice ; pourtant ce sont essentiellement sur les grâces royales que se fondent les principales études ${ }^{3}$. Certes, les lettres bourguignonnes font régulièrement l'objet de recherches, toutefois celles-ci portent le plus souvent sur les territoires septentrionaux gouvernés par les ducs Valois à la fin du Moyen Âge ${ }^{4}$. La thèse réalisée a donc eu pour but de proposer une synthèse sur les pratiques de la grâce criminelle dans l'ensemble de l'espace bourguignon, depuis le XIV ${ }^{\mathrm{e}}$ siècle jusqu'à la mort de Charles le Téméraire $(1467-1477)^{5}$, et sur leurs évolutions, tant sur le plan des pratiques documentaires que sur celui des usages de la miséricorde ducale et son rôle dans la centralisation et la construction de la souveraineté 
princières. Les résultats obtenus permettent ainsi d'identifier des normes sociales, juridiques et politiques qui se construisent, notamment, par la pratique de la grâce ${ }^{6}$.

2 Afin d'analyser les rémissions accordées par les ducs de Bourgogne sur l'ensemble de leurs territoires, les archives des anciens ressorts des chambres des comptes de Dijon et Lille ont fait l'objet de dépouillements approfondis; en revanche, les fonds issus des archives belges et néerlandaises - anciens ressorts des chambres des comptes de Bruxelles et La Haye - n'ont pas été pris en compte ${ }^{7}$. Ces investigations ont permis de constituer un corpus de huit cents lettres de grâce, rémission, pardon, abolition et rappel de ban, délivrées par les ducs de Bourgogne, mais aussi par leurs épouses, depuis le principat d'Eudes IV (1315-1349) jusqu'à la mort de Charles le Téméraire. L'ensemble des données contenues dans ces documents a pu être traité grâce à l'établissement d'une base de données sous le logiciel FilemakerPro, constituée d'une quarantaine de critères relatifs au suppliant, à la victime, au crime, à l'expression de la grâce ou encore à la structure de la lettre. Le cadre chronologique s'est imposé en raison de la conservation des sources, aucune lettre antérieure à 1336 n'ayant été relevée, tandis que la mort du dernier duc Valois marque la fin du vaste ensemble territorial bourguignon. Par ailleurs, il était utile de s'intéresser aux pratiques de la grâce sur plusieurs territoires acquis ensuite par les ducs Valois au gré de leurs politiques matrimoniales, afin d'analyser d'éventuelles ruptures ou continuités induites par le changement dynastique. C'est pour cette raison que les quatre-vingt-deux lettres accordées par la comtesse de Bourgogne et d'Artois, Marguerite de France (1361-1382), le comte de Flandre, Louis de Male (1346-1384), et la comtesse de Bar et dame de Cassel, Yolande de Flandre (1344-1395), ont été ajoutées au corpus de cette étude, et encodées dans des bases de données fondées sur la même structure que celle des grâces bourguignonnes. Les rares documents de procédure de grâce (suppliques, entérinements) ont aussi été pris en compte, de même que les quelques actes législatifs relatifs aux pratiques de la grâce.

3 Le corpus de lettres fait cependant ressortir des lacunes chronologiques et géographiques : par exemple, le fait que l'on ne dispose que de dix grâces et rémissions octroyées par Jean sans Peur (1404-1419) ou, encore, la très forte proportion de documents accordés à des habitants du comté de Flandre (45\%). Ce dernier point peut, notamment, s'expliquer par des facteurs démographiques, ce territoire étant particulièrement urbanisé et peuplé, mais aussi et surtout par la proximité dont bénéficient alors les sujets avec les centres de pouvoirs ${ }^{8}$. Une autre limite réside dans l'importance des données inconnues au sein des lettres, telles les qualités sociales des suppliants qui apparaissent dans moins de la moitié des documents du corpus. Afin de combler certains de ces manques, les comptabilités des bailliages des duché et comté de Bourgogne ont fait l'objet de dépouillements systématiques afin de relever les sommes versées pour des amendes civiles exigées en échange de la rémission, phénomène dont l'ampleur croissante est caractéristique des pratiques de la grâce dans l'espace bourguignon. Les 382 mentions collectées parmi les quelque 500 registres conservés ont ainsi permis de combler la carence de lettres pour certaines périodes, particulièrement pour le gouvernement de Jean sans Peur, et elles ont été confrontées aux 455 autres occurrences relevées au sein de la recette générale de toutes les finances, en prêtant attention aux raisons de la présence de tels articles dans cette dernière. Les données collectées dans les comptabilités ont ensuite été traitées dans une base de données (sous FilemakerPro) constituée d'une vingtaine de critères, concernant les bénéficiaires 
des grâces, leur répartition spatiale, le vocabulaire employé par les officiers comptables pour exprimer la grâce et la nature des versements, ou encore les montants payés. L'analyse des registres de délibérations de la mairie de Dijon a également apporté des informations sur les relations entre le gouvernement urbain et le pouvoir princier au prisme des pratiques de la grâce. De la même manière, les archives de la justice échevinale ont permis de confronter plusieurs lettres de rémission aux poursuites dont les suppliants faisaient l'objet avant de bénéficier de la miséricorde ducale, en restituant parfois leurs parcours criminels sur plusieurs décennies.

La documentation étudiée permet ainsi d'établir que les ducs de Bourgogne figurent parmi les premiers princes du royaume à user de la lettre de grâce dans la première moitié du XIV siècle, au plus tard dès $1323^{9}$. Par la suite, les princes Valois adoptent rapidement le modèle de la lettre royale, si ce n'est déjà le cas dès les derniers Capétiens. On constate également que le comte Louis de Male et les comtesses Marguerite de France et Yolande de Flandre usent de la lettre de rémission dès cette période, généralement en s'inspirant de ce même modèle royal ${ }^{10}$. Il est donc patent que les ducs Valois n'importent pas les pratiques de la rémission dans l'ensemble des territoires qu'ils gouvernent, puisque celles-ci existent déjà lors de leur avènement. Il faut, en revanche, distinguer le pouvoir miséricordieux des ducs et de leurs épouses, ces dernières ayant généralement des prérogatives restreintes sur ce plan selon les principats, et le plus souvent par délégation de leur époux en son absence ${ }^{11}$. Une exception notable est constituée par les grâces accordées par Marguerite de Bavière, qui use parfois de ce pouvoir alors que Jean sans Peur est aussi présent, mais qui paraitt ne pouvoir accorder que de simples grâces et seulement pour les cas de vols ${ }^{12}$. Par ailleurs, les ducs tentent d'imposer l'exclusivité de leur droit de grâce sur l'ensemble de leurs territoires au fil du temps, avec succès dans les deux Bourgognes, dès la fin du $\mathrm{XIV}^{\mathrm{e}}$ siècle, mais de façon moins aboutie dans les territoires septentrionaux, où les relations avec certains officiers, comme le souverain bailli de Flandre, peuvent être plus tendues ${ }^{13}$.

5 L'octroi de la rémission, qu'elle soit royale ou princière, fait généralement suite à la rédaction d'une supplique de la part du requérant, produite par un clerc de chancellerie maîtrisant les pratiques administratives, qui l'aide à mettre son discours en forme afin de rendre possible l'obtention de la grâce ${ }^{14}$. La requête est ensuite examinée par le duc, la duchesse ou encore le conseil princier. Si la rémission est accordée, elle doit ensuite être mise en forme par un secrétaire de la chancellerie, qui en produit plusieurs copies, enregistrées dans la mémoire administrative princière ${ }^{15}$, mais aussi expédiées aux différents destinataires que sont le bénéficiaire de la lettre, ainsi que l'officier de justice locale chargé d'en vérifier le contenu. La rémission ne peut être effective sur le plan juridique qu'après avoir fait l'objet de cette procédure de vérification et d'entérinement, qui se déroule généralement en présence du bailli (ou sénéchal, écoutète...), du suppliant et de la partie lésée ou de ses proches dont les droits doivent être préservés ${ }^{16}$. L'officier ducal lit alors la lettre devant les parties impliquées, qui doivent confirmer ou infirmer son contenu, afin de s'assurer que la grâce n'est pas subreptice ou obreptice. Il vérifie, enfin, que la partie lésée a bien été dédommagée par le suppliant, le plus souvent par une somme d'argent, dont le montant échappe trop souvent à l'historien. Quant à la teneur du récit, sa lecture en présence des personnes impliquées dans l'affaire permet surtout de valider ce que les chercheurs qualifient de vérité judiciaire, qui fait du crime commis un acte pardonnable accepté par toutes les parties $^{17}$. L'ensemble de ces éléments forme ce que l'on peut qualifier de normes de la 
grâce princière, sur les plans administratif et judiciaire. Ils s'inspirent sans doute du modèle de la rémission royale et sont aussi en usage dans d'autres principautés, comme les duchés de Bretagne, de Bourbon ou encore de Lorraine.

$6 \quad$ L'étude de ces pratiques de l'écrit permet également d'affiner la distinction entre les multiples formes de grâces criminelles que sont la simple grâce, la rémission, le pardon, l'abolition et le rappel de ban. Il est possible de montrer qu'elle est bien effective dès la fin du Moyen Âge, et qu'elle repose au moins sur le type de sceau apposé à la lettre, qui varie de la rémission - grand sceau ducal ou sceau du secret de cire verte sur lacs de soie - au pardon - grand sceau ducal de cire rouge sur double queue - ou à la simple grâce - grand sceau ducal de cire rouge sur simple queue -, avec des conséquences sensibles sur le coût de la lettre pour son bénéficiaire. Par ailleurs, l'analyse lexicométrique effectuée selon la méthode employée par M. Nassiet ${ }^{18}$, appliquée au seul exposé des faits de la lettre de rémission, montre que le récit du suppliant s'étoffe de façon remarquable entre le principat de Philippe le Hardi (1363-1404) et celui de Philippe le Bon (1419-1467) puis de Charles le Téméraire. Le nombre de formules employées dans le dispositif du document s'accroît également au $\mathrm{Xv}^{\mathrm{e}}$ siècle, et l'on remarque qu'elles sont de plus en plus précises. En outre, les abréviations régulières des clauses au sein des lettres enregistrées soulignent l'existence de formulaires de chancellerie à l'image de celui d'Odart Morchesne, bien connu des historiens de la chancellerie royale ${ }^{19}$.

7 Les aspects judiciaires et sociaux de la lettre de rémission sont fondamentaux dans l'étude de ce type de source. La criminalité pardonnée par les ducs s'avère très diversifiée à la fin du Moyen Âge, suggérant, comme pour les rois, que peu de crimes paraissent irrémissibles. Il faut souligner l'existence d'au moins deux grâces accordées à des troupeaux de porcs, coupables d'avoir occis leur porcher, qui demeurent jusqu'à présent les uniques exemples de grâces octroyées à des animaux ${ }^{20}$. Néanmoins, l'éventail de la criminalité pardonnée se restreint dans la seconde moitié $d u \mathrm{xv}^{\mathrm{e}}$ siècle, à l'instar de ce qui se passe dans le royaume et d'autres principautés ${ }^{21}$, et comme en témoigne le fait que plus de $75 \%$ des rémissions accordées par Charles le Téméraire concernent des homicides. Cette évolution laisse penser que les princes, comme les rois, tendent désormais à se rapprocher des normes juridiques relatives aux crimes irrémissibles, en faisant montre dans le même temps d'une plus grande dureté vis-à-vis d'autres formes de crimes.

8 La diversité des qualités sociales des suppliants ne doit pas faire oublier, d'une part, que moins de la moitié du corpus permet de connaître cette information, et, d'autre part, que les proportions des diverses catégories socioprofessionnelles ne sont pas représentatives de la place qu'elles occupent réellement dans la société. Deux exemples suffisent à s'en convaincre, qui sont, d'un côté, la surreprésentation des nobles, constituant près de $10 \%$ des criminels pardonnés ${ }^{22}$, de l'autre les femmes qui sont quasi absentes des rémissions bourguignonnes. Bien que ces deux catégories se retrouvent dans des proportions similaires au sein des lettres royales, elles ne reflètent en aucun cas la place qu'elles tiennent au sein de la société et encore moins de la criminalitée ${ }^{23}$.

Par ailleurs, le suppliant doit avancer diverses circonstances et motifs pour tenter de justifier son crime et obtenir le pardon du prince. On constate, néanmoins, que ce sont les motifs ayant des fondements juridiques, tels que la bonne fama, la paix rétablie avec la partie lésée ou la légitime défense, qui sont le plus fréquemment évoqués. Ils renforcent ainsi la légitimité du duc à accorder sa grâce. De la même manière, les 
normes sociales du temps ressortent de l'utilisation qu'en font certaines catégories de suppliants, par exemple lorsque les nobles et hommes de guerre avancent fréquemment leurs états de service auprès du prince, ou quand les laboureurs évoquent leur bonne fama et la famille qu'ils ont en charge, soulignant ainsi le bon gouvernement de soi. L'analyse des dimensions judiciaire et sociale des rémissions met, en outre, au jour l'existence de disparités géographiques, dans la diversité des crimes pardonnés, mais aussi dans la situation des suppliants après leurs méfaits, ces deux éléments pouvant être liés et refléter aussi les pratiques judiciaires des divers territoires composant l'espace bourguignon. Il est encore possible de constater des inégalités entre les catégories socioprofessionnelles, particulièrement en ce qui concerne l'imposition d'une amende civile, qui affecte davantage les laboureurs et les gens de métiers que les nobles et les officiers.

Dans la mesure où l'amende civile est de plus en plus fréquemment imposée aux suppliants en compensation de la rémission sous les ducs Valois, il est fondamental de s'intéresser aux questions financières liées aux pratiques de la grâce des princes. Les lettres montrent une évolution vers la quasi-systématisation de l'amende civile, imposée dans 17,6\% des lettres de Philippe le Hardi, puis dans 59,4\% sous Philippe le Bon, et, enfin, $80,8 \%$ sous Charles le Téméraire. L'analyse lexicale des comptabilités des bailliages méridionaux de l'espace bourguignon traduit aussi l'adaptation de l'administration princière et ses évolutions, notamment avec l'apparition puis la rapide prépondérance du terme d'«amende civile » dès le principat de Jean sans Peur, au détriment du terme de "composition ${ }^{24}$. Au-delà de ces normes administratives, ce sont de véritables normes financières qui ressortent de l'examen des montants versés par les suppliants, en raison de la fréquence de certains, mais aussi de l'écart entre les différentes sommes, qui traduisent l'existence de seuils d'amendes. Quant à l'usage de ces finances de la grâce, il évolue également, du moins sur le plan structurel. L'argent des amendes civiles sert, parmi d'autres sources de revenus, aux réparations du domaine sous Philippe le Hardi en demeurant une composante des recettes baillivales jusqu'au milieu du $\mathrm{Xv}^{\mathrm{e}}$ siècle. En revanche, la création du trésor de l'Épargne par Philippe le Bon, au milieu des années 1440, centralise l'encaissement des sommes perçues pour toutes les chartes de grâce accordées par le prince, qu'il s'agisse des rémissions, abolitions, légitimations de bâtards, anoblissements, etc. ${ }^{25}$.

11 L'ensemble des évolutions évoquées témoigne des volontés politiques des ducs dans leurs pratiques de la grâce. On le remarque particulièrement dans les lettres de rémission, dans des circonstances variées telles que les rébellions urbaines aux PaysBas, l'avènement des princes au sein de nouveaux territoires, ou encore les premières entrées et fêtes pascales. Ces événements constituent autant de moments de communication politique du duc, qui montre ainsi publiquement son pouvoir judiciaire suprême en imposant peu à peu sa souverainetée ${ }^{26}$. Ils forment, avec les pratiques financières mentionnées, des marqueurs d'un gouvernement par la grâce des ducs de Bourgogne ${ }^{27}$, qu'il est pourtant difficile d'affirmer comme constitutif d'un État bourguignon, les seules lettres de rémission ne constituant qu'un modeste apport à ces problématiques.

12 L'étude des pratiques de la grâce princière à l'échelle d'une ville, en l'occurrence Dijon, permet enfin de saisir leur importance dans les relations entre l'échevinage et le duc. La mairie lutte ainsi régulièrement contre le prince tentant d'imposer sa souveraineté, bien qu'elle agisse toujours par la voie juridique, contrairement aux villes des Pays- 
$\mathrm{Bas}^{28}$. On le voit particulièrement à la fin du XIv siècle, lorsque le bailli de Dijon parvient à s'approprier le droit de vérifier et d'entériner les grâces accordées aux habitants de la ville, avec la bienveillance de Philippe le Hardi, au détriment du maire et des échevins et en dépit de leurs appels portés en parlement. On le voit encore dans la première moitié $\mathrm{du} \mathrm{xv}^{\mathrm{e}}$ siècle, quand le gouvernement urbain utilise contre Philippe le Bon certaines rémissions accordées par ses prédécesseurs et lui-même dans le cadre de conflits de juridictions ${ }^{29}$. Par ailleurs, la richesse des sources judiciaires échevinales offre la possibilité de confronter le discours de près d'une dizaine de lettres de rémission avec celui des poursuites dont les suppliants faisaient l'objet. Il en ressort parfois de très larges variations, comme le montre l'exemple du prévôt Jehan de Marnay, dont le long parcours criminel contraste singulièrement avec la bonne fama dont il se revendique, mais qui participe de la construction de sa vérité judiciaire afin d'obtenir la rémission de Philippe le Bon ${ }^{30}$.

Les normes sociales, juridiques et politiques des lettres de rémission bourguignonnes, de même que leurs évolutions montrent que les ducs suivent régulièrement le modèle royal, tant dans la structure des documents que dans l'usage qui en est fait, en développant cependant de véritables spécificités. Il conviendrait, dans le prolongement de ce travail, de poursuivre la comparaison avec les grâces d'autres princes et princesses de la fin du Moyen Âge, afin d'en souligner les points communs et différences, ainsi que leur rapport au modèle royal. Il importe également d'approfondir les recherches sur le trésor de l'Épargne, afin de mieux comprendre les enjeux et l'organisation de cette caisse personnelle, son fonctionnement quotidien et la manière dont elle s'intègre dans l'administration ducale. Enfin, les investigations sur la justice échevinale dijonnaise doivent être poursuivies, dans le but de mieux comprendre le traitement de la criminalité et sa répression par un appareil judiciaire urbain, ainsi que leurs conséquences sur les rapports entre la mairie et le duc.

Reçu : 8 septembre 2019 - Accepté : 22 octobre 2019

\section{NOTES}

1. C. GAUVARD, «De grace especial ». Crime, État et société en France à la fin du Moyen Âge, Paris, 1991, 2 vol.

2. Voir notamment: R. FIRINO, «Lettres de rémission concernant Ambleny et Pernant, 1422 ", Bulletin de la Société archéologique, historique et scientifique de Soissons, 15 (1908), p. 95-103 ; L. CÉLIER, "Les mœurs rurales en Poitou d'après les lettres de rémission ", Bulletin philologique et historique, 1958, p. 411-419 ; F. MOLARD, Esquisses de mœurs sénonaises aux XIVee et XVe siècles d'après les lettres de rémission, Sens, 1895.

3. N. ZEMON DAVIS, Pour sauver sa vie. Les récits de pardon au XVI siècle, Paris, 1988 ; J. HOAREAUDODINAU, Les atteintes verbales à l'autorité en particulier dans les lettres de rémission XIVe-XVe siècles), thèse dactylographiée, Limoges, 1994 ; P. TEXIER, La rémission au XIVe siècle. Genèse et développement, thèse d'État inédite, Limoges, 1991.

4. P. ARNADE et W. PREVENIER, Honor, Vengeance, and Social Trouble: Pardon Letters in the Burgundian Low Countries, Cornell, 2015 ; J.-M. CAUCHIES et H. DE SCHEPPER, Justice, grâce et législation. Genèse de 
l'État et moyens juridiques dans les Pays-Bas, 1200-1600, Bruxelles, 1994 ; H. DE SCHEPPER et M. VROLIJ, «La grâce princière et la composition coutumière aux Pays-Bas bourguignons, 1384-1633 ", in J. HOAREAU-DODINAU et P. TEXIER (dir.) Anthropologies juridiques. Mélanges Pierre Braun, Limoges, 1998, p. 735-759; C. PETIT-DUtAillis (éd.), Documents nouveaux sur les mœurs populaires et le droit de vengeance dans les Pays-Bas au XVe siècle, Paris, 1908 (réimp. 1975).

5. L'historiographie récente de l'espace bourguignon démontre la pertinence et la nécessité de prendre désormais en compte l'ensemble des blocs territoriaux, notamment afin de combler l'écart historiographique entre espaces septentrionaux et méridionaux. Voir notamment C. BECCHIA, Les bourgeois et le prince. Dijonnais et Lillois auprès du pouvoir bourguignon (1419-1477), Paris, 2019 ; É. LECUPPRE-DESJARDIN, Le royaume inachevé des ducs de Bourgogne, XIV -XVe siècles, Paris, 2016 ; Q. VERREYCKEN, Pour nous servir en l'armée. Le gouvernement et le pardon des gens de guerre sous Charles le Téméraire, duc de Bourgogne (1467-1477), Louvain, 2014.

6. C. Gauvard, A. Boureau et R. JACOB, "Normes, droit, rituel et pouvoir ", in O. G. oeXLE et J.-C. schмiтt (dir.), Les tendances actuelles de l'histoire du Moyen Âge en France et en Allemagne, Paris, 2002, p. 460-482; B. GARNOT (dir.), Normes juridiques et pratiques judiciaires du Moyen Âge à l'époque contemporaine, Dijon, 2007.

7. La documentation réunie provient ainsi de fonds conservés aux archives départementales de la Côte-d'Or [désormais ADCO], du Nord [désormais ADN] et du Doubs, des archives municipales de Dijon, Beaune, Besançon, de la bibliothèque municipale de Dijon ainsi que des Archives nationales.

8. M. NASSIET et A. MUSIN, «L'exercice de la rémission et la construction étatique (France, PaysBas) », Revue historique, 661 (2012), p. 3-26.

9. ADCO, B 11466, mars 1323.

10. B. GRÉVIN, «L'influence des modèles italiens du XIII ${ }^{\mathrm{e}}$ siècle sur le style de la chancellerie royale et des chancelleries princières françaises aux XIV et Xv $v^{\mathrm{e}}$ siècles ", in G. CASTELNUOvo et O. MATTÉONI (dir.), «De part et d'autre des Alpes» (II). Chancelleries et chanceliers des princes à la fin du Moyen Âge, Chambéry, 2011, p. 111-136.

11. M. sомmÉ, «Les délégations de pouvoir à la duchesse de Bourgogne Isabelle de Portugal au milieu du XV $v^{e}$ siècle ", in Les princes et le pouvoir au Moyen Âge. XXIII ${ }^{e}$ Congrès de la SHMESP, Brest (mai 1992), Paris, 1993, p. 285-301.

12. Sur les pouvoirs de Marguerite de Bavière en absence du duc, voir A. MARCHANDISSE, «Le pouvoir de Marguerite de Bavière, duchesse de Bourgogne. Une esquisse ", in E. BOUSMAR, J. DUMONT, A. MARCHANDISSE et B. SCHNERB (dir.), Femmes de pouvoir, femmes politiques durant les derniers siècles du Moyen Âge et au cours de la première Renaissance, Bruxelles, 2012, p. 493-506.

13. J. VAN ROMPAEY, Het grafelijk baljuwsambt in Vlaanderen tijdens de Boergondische periode, Bruxelles, 1967.

14. P. TEXIER, «La part de l'ombre dans la rémission. Remarques sur le rôle du clerc dans la rédaction de la requête en rémission ", in J. PÉRICARD (dir.), La part de l'ombre : artisans du pouvoir et arbitres des rapports sociaux (VIII ${ }^{e}-\mathrm{XV}^{e}$ siècles), Limoges, 2014, p. 183-206.

15. R. SCHEURER, "L'enregistrement à la chancellerie de France au cours du $x v^{\mathrm{e}}$ siècle", Bibliothèque de l'École des chartes, 120 (1962), p. 104-129.

16. P. TEXIER, «Qui parlera pour le mort? Les droits de la partie offensée dans les actes de grâce pénale (XIII ${ }^{\mathrm{e}}-\mathrm{XV}$ e siècles) », in J. HOAREAU-DODINAU, G. MÉTAIRIE et P. TEXIER (dir.), Procéder. Pas d'action, pas de droit ou pas de droit, pas d'action, Limoges, 2006, p. 139-153.

17. N. ZEMON DAVIS, Pour sauver sa vie..., op. cit., p. 50-54.

18. M. NASSIET, «Le récit de crime rémissible au XVI ${ }^{\mathrm{e}}$ siècle », in L. FAGGION et C. REGINA (dir.), Récit et justice : France, Italie, Espagne, XIV ${ }^{e}$-XIX ${ }^{e}$ siècles, Aix-en-Provence, 2014, p. 143-160.

19. O. GUYOTJEANNIN et S. LUSIGNAN (éd.), Le formulaire d'Odart Morchesne, Paris, 2005. 
20. ADCO, B 10440, fol. 6v-7v; B 2761, fol. $5 \mathrm{v}$.

21. C. GAUVARD, «La justice pénale du roi de France à la fin du Moyen Âge ", in X. ROusseauX et R. LÉVY (dir.), Le pénal dans tous ses États. Justice, États et sociétés en Europe (XII ${ }^{e}-\mathrm{XX}{ }^{e}$ siècles), Bruxelles, 1997, p. 104-105.

22. R. BEAULANT, «La représentation de la noblesse au sein des lettres de rémission des ducs de Bourgogne ", Publications du Centre européen d'études bourguignonnes, 58 (2018) [Autour des Chalon et de la noblesse en pays bourguignon (XIV ${ }^{e}-X V I^{e}$ siècles)], p. 103-118.

23. C. GAUVARD, « De grace especial ». Crime..., op. cit., t. 1, p. 74 et 300.

24. Voir notamment B. DAUVEN ET A. MUSIN, «Composition et rémission: deux modalités complémentaires du droit de grâce? ", in X. RousSEAUX, M.-A. BOURGUIGNON et B. DAUVEN (dir.), Amender, sanctionner et punir. Histoire de la peine du Moyen Âge au XXe siècle, Louvain, 2012, p. 49-59.

25. Р. КАUСН, «Le trésor de l'Épargne, création de Philippe le Bon », Revue belge de philologie et d'histoire, 11 (1932), p. 703-719.

26. Sur la communication politique des ducs, voir É. LECUPPRE-DESJARDIN, La ville des cérémonies. Essai sur la communication politique dans les anciens Pays-Bas bourguignons, Turnhout, 2004.

27. P. PRÉTOU, « Les lettres de grâce des rois de France au Moyen Âge », in Criminocorpus [en ligne], Les sources de la recherche, Articles, 2018 ; C. GAUVARD, « Le roi de France et le gouvernement par la grâce à la fin du Moyen Âge. Genèse et développement d'une politique judiciaire ", in H. MILLET (dir.), Suppliques et requêtes. Le gouvernement par la grâce en Occident (XII ${ }^{e}-X V^{e}$ siècle), Rome, 2003, p. 371-404.

28. C. BERTUCAT, «La juridiction municipale de Dijon. Son étendue », Revue bourguignonne, 21/2 (1911), p. 87-231 ; B. SCHNERB, L'État bourguignon, 1363-1477, Paris, 1999, p. 395-405.

29. Archives municipales de Dijon, $C 4$.

30. ADN, B 1692, fol. 19v-21; ADCO, série B II 360. Sur la vérité judiciaire, voir J. THÉRY, « Fama : l'opinion publique comme preuve judiciaire ", in B. LEMESLE (dir.), La preuve en justice de l'Antiquité à nos jours, Rennes, 2003, p. 140 ; C. LEVELEUX, «Droit et vérité. Le point de vue de la doctrine médiévale ( $\mathrm{XII}^{\mathrm{e}}-\mathrm{XV}^{\mathrm{e}}$ siècle) ou la vérité entre opinion et fiction ", in É. GAUCHER (dir.), Le vrai et le faux au Moyen Âge, Villeneuve-d'Ascq, 2005, p. 333-350.

\section{INDEX}

Mots-clés : lettres de rémission, gouvernement par la grâce, criminalité, ducs de Bourgogne, justice échevinale, normes, comptabilités 